\title{
COMPUTING INVARIANTS FOR \\ FINITELY PRESENTED NILPOTENT GROUPS
}

\author{
LEON S, SterLing
}

This thesis is concerned with computing invariants for finitely presented nilpotent groups. Two main problems are considered.

The first discusses practical methods for identifying the isomorphism type of an abelian group from a finite presentation. The classical algorithm computes the Smith normal form of the relation matrix of the presentation. A new algorithm which computes the primary invariants of the associated group rather than the torsion invariants is described. This algorithm appears more efficient for large examples. Details of the program and examples of computations also appear in Havas and Sterling [1].

The second problem centres on classifying finitely generated, torsionfree nilpotent groups of class $2:$ Canonical presentations can be given for each such group. A skew-symmetric matrix can be associated with each canonical presentation. Conditions are given in terms of these matrices for when two canonical presentations present isomorphic groups. An equivalence class of polynomials, related to the determinant of the skewsymmetric matrices, is shown to be an invariant of the group. This is only useful when the matrix has even dimension.

The conditions are used to classify torsionfree nilpotent groups with cyclic commutator subgroup, and groups with Hirsch number less than or equal to 6 . The results for Hirsch number equal to 6 appear new. These groups are determined by the isomorphism type of the commutator quotient group and the equivalence class of a binary quadratic form. A

Received 9 July 1981. Thesis submitted to the Australian National University November 1980. Degree approved June 1981. Supervisor: Dr M.F. Newman. 
more restricted canonical presentation is given for these groups and a test for determining when two such presentations present isomorphic groups. Nilpotent groups of class 2 with torsionfree commutator quotient group are also considered. Relational presentations can be given for each such group and a skew-symmetric matrix associated with each relational presentation. Two relational presentations present isomorphic groups when precisely the same conditions hold for the associated matrices, as for canonical presentations. Thus the classification results hold also for these groups.

Finally, practical algorithms are given for computing both canonical and relational presentations from arbitrary presentations of the respective nilpotent groups of class 2 .

\section{Reference}

[1] George Havas and Leon Sterling, "Integer matrices and abelian groups", EUROSAM '79, 431-451 (Internat. Sympos. Symbolic and Algebraic Manipulation, Marseille, 1979. Lecture Notes in Computer Scienc Science, 72. Springer-Verlag, Berlin, Heidelberg, New York, 1979).

Department of Artificial Intelligence, University of Edinburgh, Hope Park Square, Meadow Lane, Edinburgh EH8 9NW, ScotIand. 